\title{
Analysis of Periodontitis Biomarker Expression in Gingival Crevicular Fluids
}

\author{
Young Sun Hwang ${ }^{\dagger}$ \\ Department of Dental Hygiene, College of Health Science, Eulji University, Seongnam 13135, Korea
}

\begin{abstract}
Background: Periodontal disease, also known as gum disease, is a major dental inflammatory disease with a very high prevalence; it is the main cause of tooth loss. Therefore, diagnostic biomarkers that can monitor gum inflammation are important for oral healthcare. Since the gingival crevicular fluid (GCF) adequately reflects changes in the periodontal environment, they have become a target for the development of effective diagnostic biomarkers for periodontitis. In the present study, the level of the target molecules suggested as diagnostic biomarkers for periodontitis were analyzed in GCF samples collected from healthy individuals and periodontitis patients. In addition, useful targets for the diagnosis of periodontitis were evaluated.

Methods: GCF samples were collected from healthy individuals and periodontitis patients using absorbent paper points. SDS-PAGE and Coomassie staining were performed for protein analysis. The protein concentrations of GCF specimens were determined using the Bradford method. The levels of the target molecules appropriate for diagnosing periodontal disease were measured by ELISA, according to the manufacturer's protocol.

Results: The protein concentration of GCF collected from periodontitis patients was 3.72 fold higher than that in an equal volume of GCF collected from healthy individuals. ELSA analysis showed that the level of interukin-6 (IL-6), IL-8, metalloproteinases 2 (MMP-2), MMP-9, tumor necrosis factor-alpha (TNF- $\alpha$ ), azurocidin, and odontogenic ameloblast-associated protein (ODAM) were higher in the GCF samples from the periodontitis patients than in those from the healthy individuals. However, the level of $\mathrm{IL}-6$ and TNF- $\alpha$ were relatively low $(>5 \mathrm{pg} / \mathrm{ml})$. The prostaglandin $E_{2}\left(P_{G} E_{2}\right)$ levels were not significantly different between the two GCF samples.

Conclusion: These results indicate that IL-8, MMP-2, MMP-9, azurocidin, and ODAM are potentially useful diagnostic biomarkers for periodontitis; combining multiple biomarkers will improve the diagnostic accuracy of periodontitis.
\end{abstract}

Key Words: Biomarkers, Diagnosis, Gingival crevicular fluid, Oral hygiene, Periodontitis

\section{Introduction}

Gum inflammation is caused by several factors, including poor oral hygiene and systemic diseases ${ }^{1}$. Periodontitis is a chronic inflammatory disease caused by the gradual breakdown of tissues surrounding the teeth. It is a common disease that occurs in over $90 \%$ of adults and is the main cause of tooth loss. Therefore, in vitro diagnostic tests that can easily monitor the gum inflammation are likely to be effective in managing periodontal disease; hence, the development of a diagnostic biomarker that specifically responds to periodontitis is necessary. To elucidate potential diagnostic biomarkers for this disease, research has been conducted using periodontal tissue and saliva ${ }^{2,3)}$. However, diagnosis using saliva affects the accuracy of diagnosis due to the dilution and contamination of the diagnostic biomarkers. Periodontal tissue is a cellular specimen and requires a pretreatment process; therefore, further analysis is required to improve the diagnostic accuracy when using these samples. However, gingival crevicular fluid (GCF) is an appropriate target for identifying effective diagnostic 
biomarkers for periodontitis because it is located in the periodontal pocket where inflammation occurs and reflects changes in the periodontal microenvironment accurately ${ }^{4)}$.

Many researchers have attempted to identify proteinaceous diagnostic biomarkers for periodontal disease using $\mathrm{GCF}^{4-10}$. Superoxide dismutase (SOD1), apolipoprotein A-I (ApoA-I), dermcidin (DCD), L-plastin, annexin-1, azurocidin, and odontogenic ameloblast-associated protein (ODAM) have been suggested as diagnostic biomarkers based on the LC-MS/MS analysis of GCF from periodontitis patients and healthy individuals. In addition, increased production of prostaglandin $\mathrm{E}_{2} \quad\left(\mathrm{PGE}_{2}\right)$, interleukin-1beta (IL-1 $\beta$ ), IL-6, IL-8, tumor necrosis factor-alpha (TNF- $\alpha$ ), and metalloproteinases (MMPs), which are representative molecules involved in tissue destruction, are observed in periodontal disease ${ }^{11)}$. However, the levels of these biomarkers in the same sample have not yet been simultaneously compared.

In the present study, the levels of various diagnostic biomarkers for periodontitis were analyzed in GCF specimens collected from healthy individuals and periodontitis patients. Differences between the protein levels of the diagnostic biomarkers were observed in the two groups. Comparing the biomarkers suggested by many researchers in the same GCF sample will be helpful for screening effective diagnostic biomarkers ${ }^{4-11)}$. In particular, our results will be useful for optimizing the combination of multiple biomarkers to improve the diagnostic accuracy for periodontitis.

\section{Materials and Methods}

\section{Gingival crevicular fluid collection}

Sampling software ( $G^{*}$ Power Version 3.1.9.7 for Windows) was used to calculates the sample size with an alpha error of $5 \%$ and power of $95 \%$; the sample size was found to be 20 in each group. Additional samples were collected from each group to compensate for the processing errors. The GCF samples were collected from healthy individuals $(n=23)$ and periodontitis patients $(n=55)$ without systemic complications at the Seoul Hana Dental Clinic by a dentist (Seongnam, Korea). The normal group consisted of individuals with clinically healthy periodontal tissues (low scores of bleeding on probing in $<10 \%$ of the sites and no sites with a probing depth $>3$ $\mathrm{mm}$ or clinical attachment loss). The participants in the periodontitis group had teeth with probing depths of $\geq 3$ $\mathrm{mm}$ and clinical attachment loss of $\geq 3 \mathrm{~mm}$. Subjects with oral inflammation and pregnant women were excluded from this study. Written informed consent was obtained from each patient. The GCF samples were collected from the periodontal pockets around the teeth with inflamed gingival tissues using absorbent paper points (Meta Biomed, Chungju, Korea). The sample sites were dried and isolated from saliva using cotton rolls. The absorbent paper points were gently inserted into the sulcus and left in place for 30 seconds. The paper points visibly contaminated with blood were discarded. The paper points wetted with GCF were incubated in $100 \mu \mathrm{l}$ of phosphate-buffered saline with agitation for 30 minutes at $4^{\circ} \mathrm{C}$. The samples were then centrifuged at $4,000 \times \mathrm{g}$ for 10 minutes at $4^{\circ} \mathrm{C}$, and the supernatants were used for subsequent analysis.

\section{Pooling of gingival crevicular fluid samples}

The GCF samples from the healthy individuals and periodontitis patients were separately pooled to determine the amount of protein needed for enzyme-linked immunosorbent assay (ELISA) analysis.

\section{Determination of protein concentration}

The protein concentrations of the GCF specimens were quantified using the Bradford method ${ }^{12}$. A protein assay dye reagent concentrate kit was used according to the manufacturer's protocol (Bio-Rad Laboratories, Inc., Hercules, CA, USA). Bovine serum albumin was used for constructing the standard curve. Briefly, $2 \mu \mathrm{l}$ of the protein samples was mixed with $198 \mu$ l of Bradford reagent. The absorbance was measured at $595 \mathrm{~nm}$ using a microplate reader (Synergy ${ }^{\text {TM }}$ HTX Multi-Mode Microplate Reader; BioTek Instruments Inc., Winooski, VT, USA).

\section{Sodium dodecyl sulfate-polyacrylamide gel electrophoresis (SDS-PAGE) and Coomassie staining}

All reagents used for experiments were analytical grade (Sigma Aldrich Chemical Co., St. Louis, MO, USA). 
Equal volume $(10 \mu \mathrm{l})$ of the GCF specimens were mixed with sample buffer (33 mM Tris- $\mathrm{HCl}$ [pH 6.8], 24\% [w/v] glycerol, $2.1 \%$ SDS, $0.01 \%$ bromophenol blue) with $5 \%$ $\beta$-mercaptoethanol and boiled at $100^{\circ} \mathrm{C}$ for 5 minutes. The samples were then separated via $13 \%$ SDS-PAGE in 20 $\mathrm{mA}$. The gel was then stained with Coomassie Brilliant Blue solution $(0.1 \%$ [w/v] Coomassie blue R250, 20\% [v/v] methanol, and $10 \%$ [v/v] acetic acid) overnight. Protein bands were observed after destaining the gels with $10 \%$ acetic acid glacial in $40 \%$ methanol.

\section{Enzyme-linked immunosorbent assay}

The protein levels in equal amounts of the GCF specimens were examined using ELISA, according to the manufacturer's protocols. The following ELISA kits were purchased from their respective sources: Human IL-6 Quantikine ELISA kit (D6050), Human IL-8/CXCL8 Quantikine ELISA kit (D8000C), Human MMP-2 Quantikine ELISA kit (MMP200), Human MMP-9 Quantikine ELISA kit (DMP900), Prostaglandin $\mathrm{E}_{2}$ Parameter assay kit (KGE004B), and Human TNF- $\alpha$ Quantikine ELISA kit (DTA00D) (R\&D System Inc.,

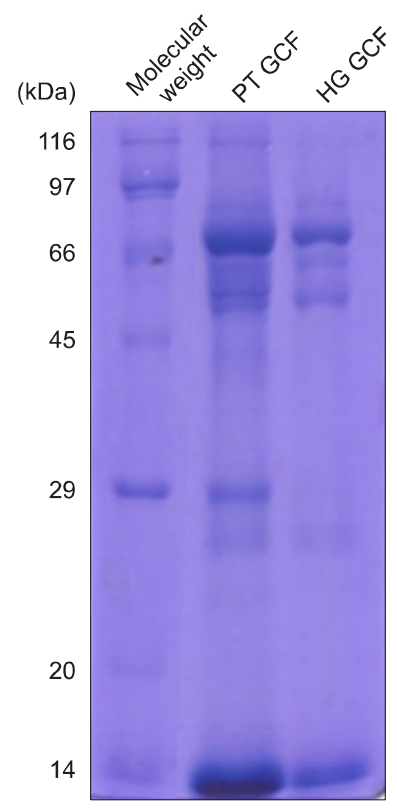

Fig. 1. Sodium dodecyl sulfate-polyacrylamide gel electrophoresis (SDS-PAGE) analysis. $10 \mu$ of gingival crevicular fluid (GCF) specimens from healthy individuals (HG) and periodontitis patients (PT) were separated by electrophoresis on 13\% acrylamide gel and stained with Coomassie Brilliant Blue. A molecular weight marker was also loaded.
Minneapolis, MN, USA); Human azurocidin ELISA kit (ab213755) (Abcam, Cambridge, UK); Human ODAM ELISA kit (A1E959) (Cusabio Biotech, Wuhan, China). The absorbance was measured using a microplate reader (BioTek Instruments Inc.).

\section{Statistical analysis}

Statistical analyses were conducted using the InStat GraphPad Prism ver. 5.01 statistical software (GraphPad Software Inc., San Diego, CA, USA). A non-parametric Mann-Whitney test was used to analyze the data from two groups. The results are presented as the mean \pm standard error of the mean. A p-value of less than 0.05 was considered statistically significant.

\section{Results}

1. Protein analysis of gingival crevicular fluid specimens from healthy individuals and periodontitis patients

The secretion volume of GCF and levels of GCF components were higher in patient with periodontal disease $^{13)}$. Equal volumes $(10 \mu \mathrm{l})$ of GCF collected from healthy individuals and periodontitis patients were analyzed by SDS-PAGE. As shown in Fig. 1, the amount of protein in equal volumes of the two samples was different. To measure the level of biomarker expression,

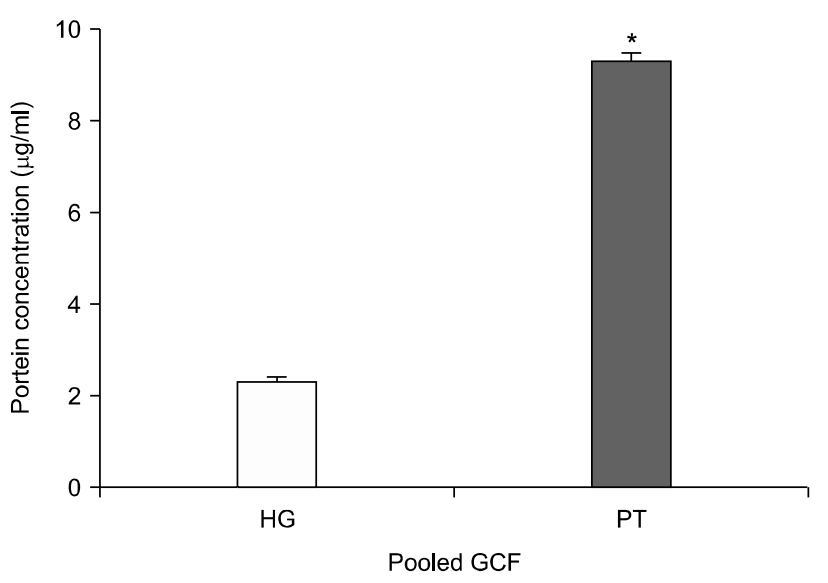

Fig. 2. Protein concentrations of pooled gingival crevicular fluid (GCF) specimens from healthy individuals (HG) and periodontitis patients (PT). The protein concentrations of GCF specimens were analyzed using the Bradford method. Absorbance was measured at $595 \mathrm{~nm}$ with a microplate reader. ${ }^{*} \mathrm{p}<0.001$ vs. HG. 
GCF samples from the healthy individuals $(n=23)$ and periodontitis patients $(n=55)$ were separately pooled to prepare the necessary amounts of protein. The protein concentration of the pooled GCF specimens were then quantified. As shown in Fig. 2, the protein concentration of the GCF samples from periodontitis patients $(9.15 \pm 0.13$ $\mu \mathrm{g} / \mathrm{ml}$ ) was 3.72 fold higher than that of the GCF samples from healthy individuals $(2.46 \pm 0.08 \mu \mathrm{g} / \mathrm{ml})$.

\section{Enzyme-linked immunosorbent assay analysis} of gingival crevicular fluid specimens from healthy individuals and periodontitis patients

The components of the GCF are susceptible to change depending on the periodontal microenvironment, making GCF an excellent source for identifying diagnostic markers for periodontitis ${ }^{4,13)}$. In this study, the levels of diagnostic biomarkers of periodontal disease reported by previous researchers were analyzed in the GCF samples form periodontitis patients and healthy individuals. As shown in Fig. 3, the level of IL-6, IL-8, MMP-2, MMP-9, TNF- $\alpha$, azurocidin, and ODAM in the GCF samples form periodontitis patients were higher than those in the GCF samples from healthy individuals. However, the $\mathrm{PGE}_{2}$ level between the two GCF samples were not significantly different. The level of IL- 6 and TNF- $\alpha$ were high in the GCF samples form periodontitis patients, but their concentration were lower $(>5 \mathrm{pg} / \mathrm{ml})$ than those of other biomarkers.

\section{Discussion}

According to the healthcare big data open system of the Health Insurance Review and Assessment Service in Korea, gingivitis and periodontal disease (K05), which has remained the second most frequent outpatient disease for the past 10 years, ranked first with regard to both the total number of patients $(1,673$ million patients) and the total amount of insurance payments $(15,321$ billion won in
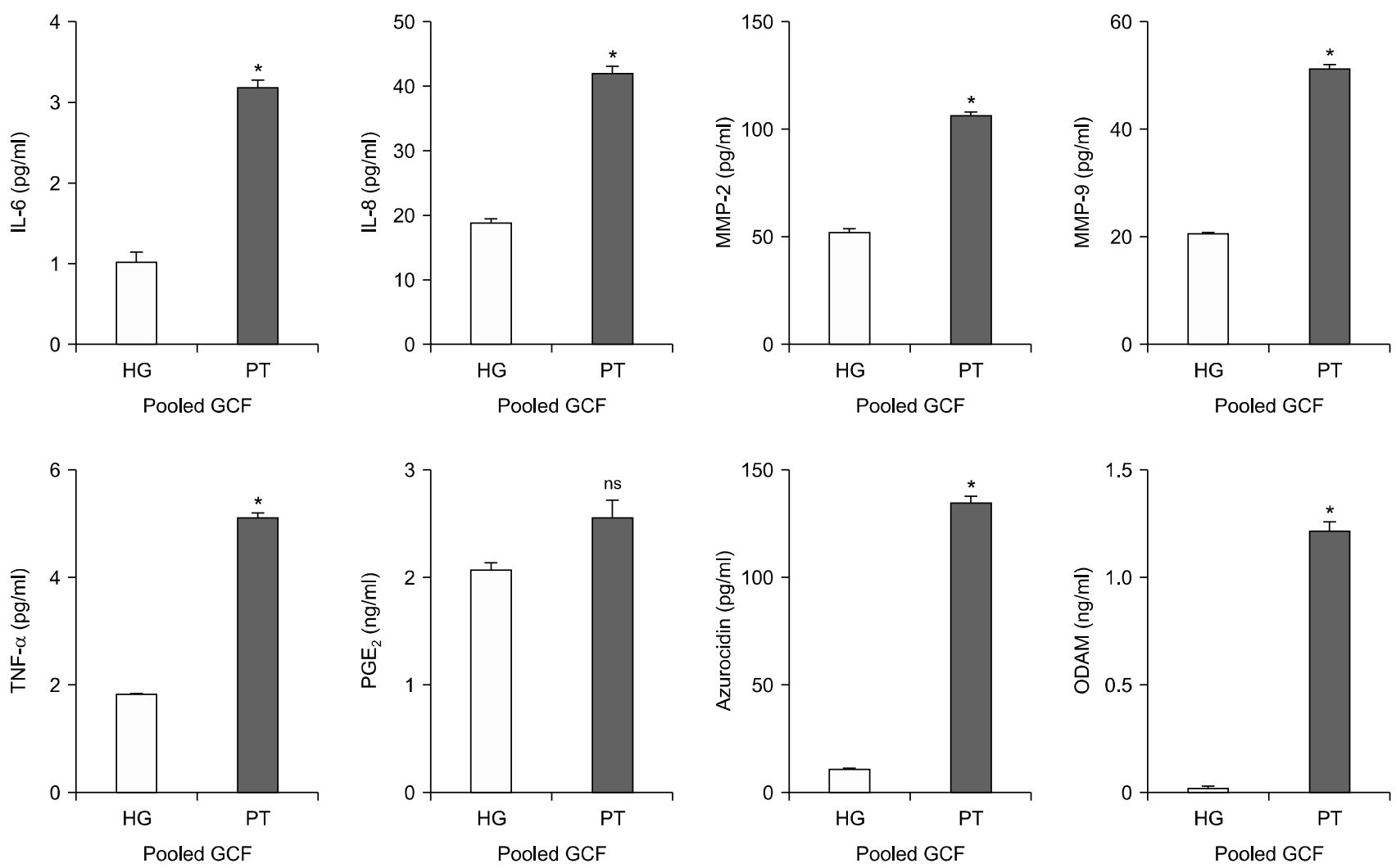

Fig. 3. Validation of various diagnostic biomarkers of periodontal disease. The protein level of IL-6, IL-8, MMP-2, MMP-9, TNF- $\alpha$, $\mathrm{PGE}_{2}$, azurocidin, and ODAM in pooled gingival crevicular fluid (GCF) specimens from healthy individuals (HG) and periodontitis patients (PT) were measured using an ELISA kit. The absorbance was measured with a microplate reader. ${ }^{*} p<0.001$ vs. HG. ns: non-specific. 
medical care benefits) in $2019^{14)}$. This is due to the high prevalence of gingivitis and periodontal disease, and also reflects an increased overall interest in oral health throughout Korea.

Since the diagnosis of periodontitis depends entirely on the dentist's opinion, an auxiliary diagnosis method for this disease is required. In vitro diagnostic test that can easily monitor gum inflammation are effective for managing periodontal disease. Therefore, the use of diagnostic kits not only reduces the number of patients with periodontitis but also improves the patient's quality of life. In particular, since non-invasive periodontal management is required after dental implant placement, diagnostic kits are useful. To accurately diagnose periodontitis, it is necessary to identify specific diagnostic biomarkers. SOD1, ApoA-I, DCD, L-plastin, annexin-1, azurocidin, and ODAM have been suggested as potential biomarkers via the analysis of GCF samples through various methods, including LC-MS/MS analysis ${ }^{4-10)}$. In addition, proteinaceous molecules, such as $\mathrm{PGE}_{2}, \mathrm{IL}-1 \beta$, IL-6, IL-8, TNF- $\alpha$, and MMPs, which are involved in periodontal tissue destruction and inflammation, have also been suggested as potential biomarkers for periodontitis ${ }^{11}$.

The balance between periodontal health and disease depends on many factors. When the production of inflammatory cytokines, such as IL-1, IL-6, TNF- $\alpha, \mathrm{PGE}_{2}$, and MMP, increases, periodontal destruction occurs ${ }^{11)}$. The production of anti-inflammatory cytokines such as IL-4, IL-10, interleukin-1 receptor (IL-1R) antagonist and tissue inhibitors of matrix metalloproteinases (TIMPs) were increased in healthy periodontium ${ }^{15}$. IL- $1 \beta$ is directly involved in processes associated with periodontal destruction, including the loss of periodontal attachment, collagen destruction, and alveolar bone resorption. The IL-1 $\beta$ levels are high in patients with periodontal disease; these levels decreases significantly after treatment ${ }^{16}$. The secretion of IL-6 is increased by inflammatory stimuli, such as IL-1 and TNF- $\alpha^{17}$. IL-6 also promotes osteoclast differentiation, resulting in bone loss ${ }^{17}$. The gene expression and protein levels of IL-8 are higher in gingival tissues of chronic periodontitis patients than in normal individuals with healthy periodontal tissues ${ }^{18)}$. The levels of MMP-2 and MMP-9 are also elevated in patients with periodontitis; these MMPs participate in tissue destruction ${ }^{19)}$. TNF- $\alpha$ increases cell permeability by regulating the expression of adhesion molecules in vascular endothelial cells and upregulates the expression of collagenases, $\mathrm{PGE}_{2}$, and proteolytic enzymes, leading to the loss of adhesion of periodontal tissues and osteoclast differentiation ${ }^{20)}$. $\mathrm{PGE}_{2}$ has been reported to be a marker for the progression and severity of periodontal disease because its expression is increased in inflamed gingival tissues ${ }^{21)}$. Azurocidin, a member of the serine protease family, is upregulated in periodontitis patients ${ }^{9}$. ODAM is involved in the adhesion of the junctional epithelium to the tooth surface and is released into the gingival crevice following the loss of adhesion of periodontal tissues during the progression of periodontitis ${ }^{10)}$. In this study, the levels of IL-6, IL-8, MMP-2, MMP-9, TNF- $\alpha$, azurocidin, and ODAM were higher in GCF samples from periodontitis patients than in those from healthy individuals. This is consistent with the results reported in previous studies. Using these biomarkers as diagnostic targets could be effective for the diagnosing of periodontitis. However, a combination of multiple biomarkers could improve diagnostic accuracy. Therefore, further studies on the diagnostic accuracy of single and multiple markers is required.

The levels of IL- 6 and TNF- $\alpha$, and PGE $_{2}$, which are commonly used as indicators to identify inflammation, in the GCF samples from periodontitis patients were either very low or did not show any significant changes, compared to those in the GCF samples from healthy individuals. Further verification of these findings is required, but a biomarker with a low concentration or whose levels show only a small change between samples can impair diagnostic accuracy. Even though it is considered an effective biomarker, the protein level of azurocidin was decreased to a great degree in severe chronic periodontitis than in chronic gingivitis and moderate periodontitis ${ }^{9}$. The levels of ODAM were also found to have a broad range among individuals ${ }^{10}$. Therefore, the validation of IL- 6 and TNF- $\alpha$, and $\mathrm{PGE}_{2}$ as biomarkers is required at differential stages during the progression of periodontitis in a large cohort.

Biomarkers are substances whose levels can be 
objectively measured as indicators of normal biological conditions, pathological progression, or pharmacological response to treatment. They can be used in diagnosing and categorizing the stages of a disease, determining prognosis, and predict clinical responses, among other purposes. Neutrophils, lymphocytes, macrophages, serum, inflammation-related molecules, antibodies, and bacteriaderived components are present in the $\mathrm{GCF}^{4}$. Therefore, the GCF has a defense mechanism to efficiently control inflammation in the surrounding tissues compared to saliva. When inflammation is increased in the surrounding tissues, the permeability of the blood vessels increases, thereby increasing the secretion of GCF, which can restore the homeostasis of the periodontal pockets. In this study, GCF was evaluated the biomarkers of periodontitis instead of saliva and periodontal tissues. The secretion of GCF is increased following inflammation, but the volume of GCF secreted and protein concentration of GCF are limited. The SDS-PAGE analysis showed that the amount of protein in equal volume of GCF from healthy individuals and periodontitis patients were different. To simultaneously analyze multiple biomarkers with a limited amount of GCF, the GCF samples from healthy individuals and periodontitis patients were separately pooled. Therefore, the results of this study did not analyze the levels of the diagnostic biomarkers individually. In addition, since the biomarkers were evaluated only in the GCF samples from healthy individuals and periodontitis patients, we were not able to validate the levels of these biomarker at the differential stages of periodontitis.

In this study, the levels of diagnostic biomarkers for periodontitis were analyzed in the GCF of healthy individuals and patients with periodontitis. It was confirmed that IL-8, MMP-2, MMP-9, azurocidin, and ODAM are useful in the diagnosis of periodontitis. To improve diagnostic accuracy, further studies using multiple biomarkers are necessary.

\section{Notes}

\section{Conflict of interest}

No potential conflict of interest relevant to this article was reported.

\section{Ethical approval}

Human experiment was approved by the Institutional Review Board (IRB) of the Eulji University (approval No. EU19-62).

\section{Author contributions}

Conceptualization, Experiments, Data acquisition, Formal analysis, Writing-original draft, Writing-review \& editing: Young Sun Hwang.

\section{ORCID}

Young Sun Hwang, https://orcid.org/0000-0001-7012-3434

\section{Acknowledgements}

I thank Jun su Kim (Seoul Hana Dental Clinic, Seongnam, Korea) for his expert assistance in diagnosing patients with periodontitis and collecting GCF. This research was supported by Basic Science Research Program through the National Research Foundation of Korea (NRF) funded by the Ministry of Education, Science and Technology (2018R1D1A1B07042035).

\section{References}

1. Lertpimonchai A, Rattanasiri S, Arj-Ong Vallibhakara S, Attia J, Thakkinstian A: The association between oral hygiene and periodontitis: a systematic review and meta-analysis. Int Dent J 67: 332-343, 2017. https://doi.org/10.1111/idj.12317

2. Taba M Jr, Kinney J, Kim AS, Giannobile WV: Diagnostic biomarkers for oral and periodontal diseases. Dent Clin North Am 49: 551-571, vi, 2005. https://doi.org/10.1016/j.cden.2005.03.009

3. Taylor JJ: Protein biomarkers of periodontitis in saliva. ISRN Inflamm 2014: 593151, 2014. https://doi.org/10.1155/2014/593151

4. Barros SP, Williams R, Offenbacher S, Morelli T: Gingival crevicular fluid as a source of biomarkers for periodontitis. Periodontol 2000 70: 53-64, 2016. https://doi.org/10.1111/prd.12107

5. Carneiro LG, Nouh H, Salih E: Quantitative gingival crevicular fluid proteome in health and periodontal disease using stable isotope chemistries and mass spectrometry. J 
Clin Periodontol 41: 733-747, 2014.

https://doi.org/10.1111/jcpe.12262

6. Silva-Boghossian CM, Colombo AP, Tanaka M, Rayo C, Xiao Y, Siqueira WL: Quantitative proteomic analysis of gingival crevicular fluid in different periodontal conditions. PLoS One 8: e75898, 2013.

https://doi.org/10.1371/journal.pone.0075898

7. Tsuchida S, Satoh M, Umemura H, et al.: Proteomic analysis of gingival crevicular fluid for discovery of novel periodontal disease markers. Proteomics 12: 2190-2202, 2012. https://doi.org/10.1002/pmic.201100655

8. Bostanci N, Heywood W, Mills K, Parkar M, Nibali L, Donos $\mathrm{N}$ : Application of label-free absolute quantitative proteomics in human gingival crevicular fluid by LC/MS E (gingival exudatome). J Proteome Res 9: 2191-2199, 2010. https://doi.org/10.1021/pr900941z

9. Choi YJ, Heo SH, Lee JM, Cho JY: Identification of azurocidin as a potential periodontitis biomarker by a proteomic analysis of gingival crevicular fluid. Proteome Sci 9: 42, 2011. https://doi.org/10.1186/1477-5956-9-42

10. Lee HK, Kim SJ, Kim YH, Ko Y, Ji S, Park JC: Odontogenic ameloblast-associated protein (ODAM) in gingival crevicular fluid for site-specific diagnostic value of periodontitis: a pilot study. BMC Oral Health 18: 148, 2018. https://doi.org/10.1186/s12903-018-0609-0

11. Ramadan DE, Hariyani N, Indrawati R, Ridwan RD, Diyatri I: Cytokines and chemokines in periodontitis. Eur J Dent 14: 483-495, 2020. https://doi.org/10.1055/s-0040-1712718

12. Bradford MM: A rapid and sensitive method for the quantitation of microgram quantities of protein utilizing the principle of protein-dye binding. Anal Biochem 72: 248-254, 1976. https://doi.org/10.1006/abio.1976.9999

13. Gupta S, Chhina S, Arora SA: A systematic review of biomarkers of gingival crevicular fluid: their predictive role in diagnosis of periodontal disease status. J Oral Biol Craniofac Res 8: 98-104, 2018.

https://doi.org/10.1016/j.jobcr.2018.02.002

14. Healthcare Bigdata Hub, Health Insurance Review and Assessment Service in Korea: Statistics of frequent disease in 2019. Retrieved January 24, 2021, from http://opendata. hira.or.kr/op/opc/olapHifrqSickInfo.do.

15. Kayal RA: The role of osteoimmunology in periodontal disease. Biomed Res Int 2013: 639368, 2013. https://doi.org/10.1155/2013/639368

16. Cheng $\mathrm{R}, \mathrm{Wu} \mathrm{Z}$, Li M, Shao M, Hu T: Interleukin-1 $\beta$ is a potential therapeutic target for periodontitis: a narrative review. Int J Oral Sci 12: 2, 2020. https://doi.org/10.1038/s41368-019-0068-8

17. Tanaka T, Narazaki M, Kishimoto T: IL-6 in inflammation, immunity, and disease. Cold Spring Harb Perspect Biol 6: a016295, 2014.

https://doi.org/10.1101/cshperspect.a016295

18. Finoti LS, Nepomuceno R, Pigossi SC, Corbi SC, Secolin R, Scarel-Caminaga RM: Association between interleukin-8 levels and chronic periodontal disease: a PRISMA-compliant systematic review and meta-analysis. Medicine (Baltimore) 96: e6932, 2017. https://doi.org/10.1097/MD.0000000000006932

19. Mäkelä $M$, Salo $T$, Uitto VJ, Larjava $H$ : Matrix metalloproteinases (MMP-2 and MMP-9) of the oral cavity: cellular origin and relationship to periodontal status. J Dent Res 73: 1397-1406, 1994. https://doi.org/10.1177/00220345940730080201

20. Sprague AH, Khalil RA: Inflammatory cytokines in vascular dysfunction and vascular disease. Biochem Pharmacol 78: 539-552, 2009.

https://doi.org/10.1016/j.bcp.2009.04.029

21. Båge T, Kats A, Lopez BS, et al.: Expression of prostaglandin E synthases in periodontitis immunolocalization and cellular regulation. Am J Pathol 178: 1676-1688, 2011. https://doi.org/10.1016/j.ajpath.2010.12.048 